\title{
ELLIPTIC EQUATIONS \\ WITH LIMITING SOBOLEV EXPONENT: THE IMPACT OF THE GREEN'S FUNCTION
}

\author{
OLIVIER REY \\ Centre de Mathématiques, Ecole Polytechnique \\ F-91128 Palaiseau, France
}

In this paper, I propose to describe some results obtained in the last few years concerning nonlinear elliptic equations, giving rise to variational problems with lack of compactness. By lack of compactness, we mean that the functionals that we consider do not satisfy the Palais-Smale condition, i.e. there exist sequences along which the functional remains bounded, its gradient goes to zero, and which do not converge. In fact, the Palais-Smale condition (PS) is not an ideal tool in variational theory. If we consider two level sets of a functional and if we try to deform one of them onto the other by using the gradient flow, two types of obstruction may occur: a stop at a critical point:

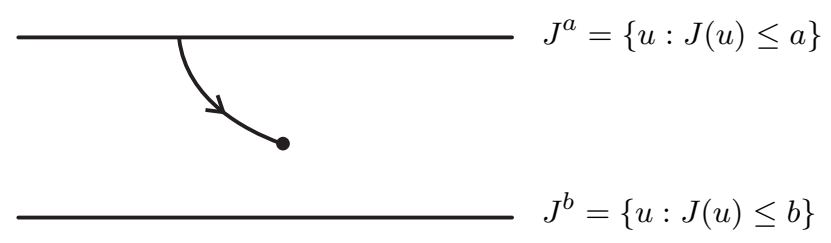

or a critical point at infinity:

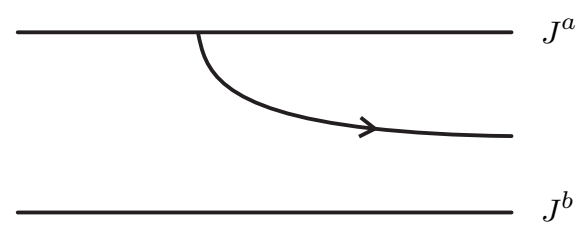

i.e. an orbit of the gradient along which the functional remains bounded, the gradient goes to zero, and the orbit does not converge. This second case is 
excluded by the Palais-Smale condition which, however, also forbids the situation

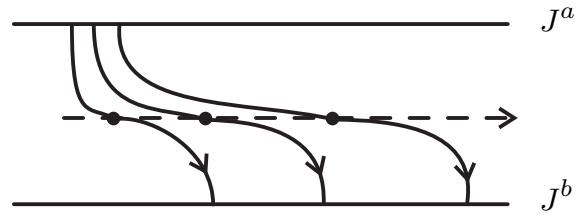

which does not present any danger from the variational viewpoint. Thus it appears that the important fact is whether or not the Palais-Smale condition is satisfied along the flow lines, i.e. whether critical points at infinity exist or not. Answering this question may be far more difficult than to determine the failure of the PalaisSmale condition. Nevertheless, if one looks for solutions to the variational problem by studying the difference of topology between the level sets of the functional, one has to identify the critical points at infinity in order to compute their contribution to the topological changes [4].

In the following, we consider more precisely nonlinear elliptic equations of the form

$$
\left\{\begin{array}{l}
-\Delta u=u^{p}+a(x) u, \quad u>0 \text { on } \Omega, \\
u=0 \text { on } \partial \Omega,
\end{array}\right.
$$

where $\Omega$ is a smooth and bounded domain in $\mathbb{R}^{N}, N \geq 3, a(x)$ is a given function and $p=(N+2) /(N-2)$. The interest in this type of equations comes from the Yamabe problem (see [33], [18] for example), which corresponds to the special case $a(x)=-\frac{N-2}{4(N-1)} R(x)$, where $R(x)$ is the scalar curvature of a Riemaniann manifold $M$ of dimension $N$, without boundary:

$$
-\Delta u=u^{p}-\frac{N-2}{4(N-1)} R(x) u, \quad u>0 \quad \text { on } M .
$$

However, Problem (1) came to an autonomous life which did not cease with the solution of the Yamabe conjecture by T. Aubin [2], N. Trudinger [32] and R. Schoen [28]. Despite its inoffensive aspect, the equation provides a whole world of ideas and questions, some of them already solved, some others remaining open. The special nature of the problem appears when we consider it from the variational viewpoint. Let us denote by

$$
J_{1}(u)=\frac{1}{2} \int_{\Omega}|\nabla u|^{2}-\frac{1}{p+1} \int_{\Omega}|u|^{p+1}-\frac{1}{2} \int_{\Omega} a(x) u^{2}, \quad \forall u \in H_{0}^{1}(\Omega),
$$

or

$$
J_{2}(u)=\frac{\int_{\Omega}|\nabla u|^{2}-\int_{\Omega} a(x) u^{2}}{\left(\int_{\Omega}|u|^{p+1}\right)^{2 /(p+1)}}, \quad \forall u \in H_{0}^{1}(\Omega)-\{0\},
$$

functionals whose (positive) critical points are solutions to (1) (up to a multiplicative constant for $J_{2}$ ). 
As the embedding of $L^{p+1}(\Omega)$ into $H_{0}^{1}(\Omega)$ is not compact, the Palais-Smale condition does not hold. Nevertheless, as we are exactly in the limit case, we will dispose of a very precise description of the sequences responsible for such a failure, or equivalently of the potential critical points at infinity.

Brézis and Nirenberg [9] have shown how one can take advantage of the linear term $a(x) u$ to prove the existence of a solution to (1). They use this linear term to make $J_{2}$ smaller than a certain level under which the Palais-Smale condition holds, so that a minimization procedure is available. The blowing-up phenomenon which occurs if $a(x)$ vanishes has been analyzed in numerous papers (see [9], [10], [17], [24], [26]).

In this paper we will focus our attention on the case $a(x) \equiv 0$, which appears to be the more difficult one.

The first section will be essentially concerned with the existence results of Bahri and Coron [5], [6] related to the topology of the domain, and of Ding [14] for a contractible domain.

The second section will be devoted to a careful analysis of the subcritical approximation of (1) in order to describe and understand precisely the phenomenon of critical points at infinity which occurs in the limit case.

1. The critical case. The first contribution to the problem

$$
\left\{\begin{array}{l}
-\Delta u=u^{p}, \quad u>0 \text { on } \Omega, \\
u=0 \text { on } \partial \Omega
\end{array}\right.
$$

is a negative result due to Pokhozhaev [23], which says:

THEOREM 1. (P) has no solution under the assumption that $\Omega$ is starshaped.

Indeed, multiply the equation in $(\mathrm{P})$ by $u$ and $\sum_{i=1}^{N} x_{i} \partial u / \partial x_{i}$ respectively, and integrate by parts to obtain

$$
\int_{\Omega}|\nabla u|^{2}=\int_{\Omega} u^{p+1}
$$

and

$$
\left(1-\frac{N}{2}\right) \int_{\Omega}|\nabla u|^{2}-\frac{1}{2} \int_{\partial \Omega}(x . n)\left(\frac{\partial u}{\partial n}\right)^{2}=\left(1-\frac{N}{2}\right) \int_{\Omega} u^{p+1}
$$

so that

$$
\int_{\partial \Omega}(x . n)\left(\frac{\partial u}{\partial n}\right)^{2}=0 .
$$

Then the strong maximum principle implies that $u=0$ on $\Omega$.

More recently, Bahri and Coron proved in [6] a very important existence result, related to the topology of the domain: 
THEOREM 2. Assume that $\Omega$ has nontrivial topology, in the sense there is $l \in \mathbb{N}^{*}$ such that $H_{2 l-1}(\Omega ; \mathbb{Q}) \neq 0$ or $H_{l}(\Omega ; \mathbb{Z} / 2 \mathbb{Z}) \neq 0$. Then $(\mathrm{P})$ has a solution.

When $N=3$, every domain which is not contractible has nontrivial topology, in the sense stated above. This is not true any more for $N \geq 4$, so that in this case it remains an open question whether one can replace the assumption of Theorem 2 by $\Omega$ noncontractible. However, if $\Omega \subset \mathbb{R}^{N}$ has holes, $H_{N-1}(\Omega ; \mathbb{Z} / 2 \mathbb{Z}) \neq 0$.

R e mark. When the holes are small, one can even get a result on multiplicity of the solutions (see [25]).

Let us denote by $G$ the Green function of the operator $-\Delta$ on $\Omega$ with Dirichlet boundary conditions, and by $H$ its regular part, i.e.

$$
\begin{aligned}
& G(x, y)=\frac{1}{|x-y|^{N-2}}-H(x, y), \quad(x, y) \in \Omega \times \Omega, \\
& G=0 \text { on } \partial(\Omega \times \Omega) \text { and } H \text { is harmonic with respect to each variable. }
\end{aligned}
$$

Let $\Omega$ be a smooth and bounded domain in $\mathbb{R}^{N}$, and $a_{1}, \ldots, a_{l} l$ points in $\Omega$. For $d>0$ we denote by $\Omega_{d}$ the domain $\Omega$ with the $l$ closed balls $B\left(a_{i}, d\right)$ deleted.

For $k \in \mathbb{N}^{*}$ and $\mathbf{x}=\left(x_{1}, \ldots, x_{k}\right) \in \Omega^{k}$ we set

$$
M(\mathbf{x})=M\left(x_{1}, \ldots, x_{k}\right)=\left(m_{i j}\right)_{1 \leq i, j \leq k} \in \mathcal{M}_{k}(\mathbb{R})
$$

with

$$
m_{i i}=H\left(x_{i}, x_{i}\right), \quad m_{i j}=-G\left(x_{i}, x_{j}\right), \quad i \neq j,
$$

and we denote by

$$
\varrho(\mathbf{x})=\varrho\left(x_{1}, \ldots, x_{k}\right)
$$

the smallest eigenvalue of $M(\mathbf{x})$.

Then we state the following result:

Theorem 3. Let $k \in \mathbb{N}^{*}, k \leq l$, and $1 \leq i_{1}<\ldots<i_{k} \leq l$. Assume that $M\left(a_{i_{1}}, \ldots, a_{i_{k}}\right)$ is positive definite, i.e. $\varrho\left(a_{i_{1}}, \ldots, a_{i_{k}}\right)>0$. For $d$ small enough, there exists a solution of $(\mathrm{P})$ on $\Omega_{d}$ which concentrates around the $k$ holes $B\left(a_{i_{j}}, d\right), 1 \leq j \leq k$, as d goes to zero.

The assumption is always satisfied for $k=1$, so that we get at least $l$ solutions for $d$ small. If $M\left(a_{1}, \ldots, a_{l}\right)>0$, the number of solutions is at least $2^{l}-1$.

In contrast with Theorem 2, W. Y. Ding gave in [14] an example of a contractible domain on which $(\mathrm{P})$ has a solution (for such an example, see also Dancer [12]). Such a domain is constructed as a perturbation of an annulus $A_{s}=\left\{x \in \mathbb{R}^{N}: 0<s<|x|<1\right\}$, with a thin cylinder $C_{\varepsilon}=\left\{x=\left(x_{1}, x^{\prime}\right) \in\right.$ $\left.\mathbb{R} \times \mathbb{R}^{N-1}: 0 \leq x_{1} \leq 1,\left|x^{\prime}\right|<\varepsilon\right\}$ deleted. The method used follows essentially the same idea as Coron in [11], where it was proved that $(\mathrm{P})$ has a solution provided that $\Omega$ has a small hole.

This example is interesting because it shows that just as the topology may play a role in the existence of solutions to $(\mathrm{P})$, so does the geometry of the domain. 
In fact, we believe that the good condition for existence of solutions to $(\mathrm{P})$ should be expressed as some properties of the Green function and its regular part on $\Omega$ - such that some topological conditions as in Theorem 2 make them satisfied, or some geometric conditions as in Theorem 1 make them fail. We will give some results in this direction in the next section.

Let us now sketch the proof of Theorem 2 .

All this proof is carried out under the assumption that $(\mathrm{P})$ has no solution, until we get a contradiction.

We consider on $H_{0}^{1}(\Omega)$ the functional

$$
J(u)=\frac{1}{2} \int_{\Omega}|\nabla u|^{2}-\frac{1}{p+1} \int_{\Omega}\left(u^{+}\right)^{p+1}
$$

whose nonzero critical points are solutions to $(\mathrm{P})$. First, we show that the PalaisSmale condition is satisfied for $J$ except at the levels $c_{k}=k S^{N / 2} / N, k \in \mathbb{N}^{*}$, where $S$ denotes the Sobolev constant,

$$
S=\inf _{u \in H_{0}^{1}(\Omega)-\{0\}} \frac{\int_{\Omega}|\nabla u|^{2}}{\left(\int_{\Omega}|u|^{p+1}\right)^{2 /(p+1)}} .
$$

$S$ is independent of $\Omega \subset \mathbb{R}^{N}$, and is never achieved for $\Omega$ bounded, whereas it is on $\mathbb{R}^{N}$ for all the functions

$$
\delta_{\lambda, x}(y)=\frac{\lambda^{(N-2) / 2}}{\left(1+\lambda^{2}|y-x|^{2}\right)^{(N-2) / 2}}, \quad \lambda \in \mathbb{R}_{+}^{*}, x \in \mathbb{R}^{N},
$$

which are the only minimizers ([1], [31], [19]), up to a multiplicative constant. The $\delta_{\lambda, x}$ 's are solutions on $\mathbb{R}^{N}$ of the equation

$$
-\Delta \delta=N(N-2) \delta^{p} .
$$

Moreover, these functions are the only positive solutions of (10) with $\nabla \delta \in$ $L^{2}\left(\mathbb{R}^{N}\right)$ and $\delta \in L^{p+1}\left(\mathbb{R}^{N}\right)$ (see [22], [16], [15]; this does not hold without the assumption of positivity for solutions to $-\Delta U=N(N-2)|U|^{p-1} U$ on $\mathbb{R}^{N}$, see [13]).

It is easy to see that (PS) fails at level $c_{k}$. Indeed, consider any sequence $\left(x_{i}^{n}\right)$ in $\Omega,\left(\lambda_{i}^{n}\right)$ in $\mathbb{R}_{+}^{*},\left(\alpha_{i}^{n}\right)$ in $\mathbb{R}, 1 \leq i \leq k$, such that

$$
\begin{gathered}
\alpha_{i}^{n} \rightarrow \alpha_{N}=(N(N-2))^{(N-2) / 4,} \\
\quad \lambda_{i}^{n} d\left(x_{i}^{n}, \partial \Omega\right) \rightarrow+\infty, \\
\frac{\lambda_{i}^{n}}{\lambda_{j}^{n}}+\frac{\lambda_{j}^{n}}{\lambda_{i}^{n}}+\lambda_{i}^{n} \lambda_{j}^{n}\left|x_{i}^{n}-x_{j}^{n}\right|^{2} \rightarrow+\infty, \quad i \neq j,
\end{gathered}
$$

as $n \rightarrow+\infty$.

Let us denote by $P \delta_{\lambda, x}$ the projection of $\delta_{\lambda, x}$ onto $H_{0}^{1}(\Omega)$, defined by

$$
\left\{\begin{array}{l}
\Delta P \delta_{\lambda, x}=\Delta \delta_{\lambda, x} \quad \text { on } \Omega, \\
P \delta_{\lambda, x}=0 \quad \text { on } \partial \Omega .
\end{array}\right.
$$


Then any sequence of functions

$$
u_{n}=\sum_{i=1}^{k} \alpha_{i}^{n} P \delta_{\lambda_{i}^{n}, x_{i}^{n}}
$$

where $\alpha_{i}^{n}, \lambda_{i}^{n}, x_{i}^{n}$ are chosen as stated above, satisfies

$$
J\left(u_{n}\right) \rightarrow c_{k}, \quad J^{\prime}\left(u_{n}\right) \rightarrow 0 .
$$

As $\left(u_{n}\right)$ is not relatively compact in $H_{0}^{1}(\Omega)$ (since for example $\left|\nabla u_{n}\right|^{2} \rightarrow$ $S^{N / 2} \sum_{i=1}^{k} \delta_{x_{i}}$ in the sense of measures, with $\delta_{x_{i}}$ the Dirac mass at $x_{i}=\lim x_{i}^{n}$ $\in \bar{\Omega})$, (PS) fails at level $c_{k}$.

Condition (12) means that the "boundary effect" is negligible compared to the "concentration effect", i.e. at the first order $P \delta_{\lambda_{i}^{n}, x_{i}^{n}}$ behaves as $\delta_{\lambda_{i}^{n}, x_{i}^{n}}$. Condition (13) means that the "interaction effect" between the $P \delta_{\lambda_{i}^{n}, x_{i}^{n}}$ is also negligible. Condition (12) is related to the fact that

$$
-\Delta\left(\alpha_{N} \delta\right)=\left(\alpha_{N} \delta\right)^{p} \quad \text { on } \mathbb{R}^{N} \text {. }
$$

Then (11)-(13) imply that $u_{n}$ is "almost" a solution of $(3)$, so that $J^{\prime}\left(u_{n}\right) \rightarrow 0$, and

$$
J\left(u_{n}\right) \sim k\left(\frac{1}{2} \int_{\mathbb{R}^{N}}|\nabla \delta|^{2}-\frac{1}{p+1} \int_{\mathbb{R}^{N}} \delta^{p+1}\right)=k \frac{S^{N / 2}}{N} .
$$

(For extensive computations of $J\left(u_{n}\right)$ and $J^{\prime}\left(u_{n}\right)$, see [3], [23].)

If $u_{0}$ were a solution to $(\mathrm{P})$, the sequence $u_{0}+\sum_{i=1}^{k} \alpha_{i}^{n} P \delta_{\lambda_{i}^{n}, x_{i}^{n}}$ would show that (PS) fails at the level $J\left(u_{0}\right)+c_{k}$. In fact, (PS) fails exactly at the levels $\sigma+k S^{N / 2} / N, k \in \mathbb{N}^{*}$, where $\sigma$ is any critical value of $J$. Under our assumption that (P) has no solution, it remains to prove that (PS) holds for every level $c \neq c_{k}$, $k \in \mathbb{N}^{*}$

Assume first that $c<c_{1}=S^{N / 2} / N$, and let $\left(u_{n}\right)$ be a sequence such that $J\left(u_{n}\right) \rightarrow c, J^{\prime}\left(u_{n}\right) \rightarrow 0$ as $n \rightarrow+\infty$. This means

$$
\begin{aligned}
& \frac{1}{2} \int_{\Omega}\left|\nabla u_{n}\right|^{2}-\frac{1}{p+1} \int_{\Omega}\left(u_{n}^{+}\right)^{p+1}=c+o(1), \\
& -\Delta u_{n}=\left(u_{n}^{+}\right)^{p}+f_{n} \quad \text { with } f_{n} \rightarrow 0 \text { in } H^{-1} .
\end{aligned}
$$

Multiplying (17) by $u_{n}$ and integrating on $\Omega$, we get

$$
\int_{\Omega}\left|\nabla u_{n}\right|^{2}=\int_{\Omega}\left(u_{n}^{+}\right)^{p+1}+o\left(\left|u_{n}\right|_{H_{0}^{1}}\right) .
$$

Therefore we deduce from (16) that

$$
\int_{\Omega}\left|\nabla u_{n}\right|^{2}=N c+o(1), \quad \int_{\Omega}\left(u_{n}^{+}\right)^{p+1}=N c+o(1) .
$$

Then Sobolev's inequality yields

$$
N c \geq S(N c)^{2 /(p+1)}
$$


so that either $c=0$ or $c \geq S^{N / 2} / N$, and the assumption $c<S^{N / 2} / N$ implies that $u_{n} \rightarrow 0$ in $H_{0}^{1}$.

Suppose now that $J^{\prime}\left(u_{n}\right) \rightarrow 0$ and $J\left(u_{n}\right) \rightarrow c \geq S^{N / 2} / N$. We are going to show that there exist $k \in \mathbb{N}^{*}$ and sequences $\left(\lambda_{i}^{n}\right)$ and $\left(x_{i}^{n}\right)$ satisfying (12), (13) such that

$$
\left|u_{n}-\sum_{i=1}^{k} \alpha_{N} P \delta_{\lambda_{i}^{n}, x_{i}^{n}}\right|_{H_{0}^{1}} \rightarrow 0
$$

and thus $c=c_{k}$. As previously, we deduce from (16) and (17) that $\left(u_{n}\right)$ is bounded in $H_{0}^{1}$, so that $u_{n}$ goes weakly to a limit which is zero by the assumption that $(\mathrm{P})$ has no solution. However, $u_{n}$ does not converge strongly to zero. We consider as in [21], [8] the concentration function

$$
Q_{n}(t)=\sup _{x \in \Omega} \int_{x+t B(0,1)}\left(u_{n}^{+}\right)^{p+1}, \quad t \in \mathbb{R}^{+}
$$

( $u_{n}$ is extended by 0 outside $\Omega$ ). The $Q_{n}$ 's are increasing functions, and if we assume that $0 \in \Omega$ and $\Omega \subset B(0, R)$, we have

$$
Q_{n}(R)=\left|u_{n}^{+}\right|_{p+1}^{p+1}=N c+o(1) .
$$

If $Q_{n}(R)$ goes to zero, then (18) implies that $u_{n}$ (or its subsequence) goes to zero in $H_{0}^{1}$, a contradiction. Thus, there exists $\beta>0$ such that $Q_{n}(R) \geq \beta$ for all $n$. We choose $\nu$ such that

$$
0<\nu<\min \left(\beta, S^{N / 2}\right) .
$$

There exist $\varepsilon_{n}, 0<\varepsilon_{n}<R$, and $a_{n} \in \bar{\Omega}$ such that

$$
Q_{n}\left(\varepsilon_{n}\right)=\int_{a_{n}+\varepsilon_{n} B(0,1)}\left(u_{n}^{+}\right)^{p+1}=\nu .
$$

Then we set

$$
v_{n}: \mathbb{R}^{N} \rightarrow \mathbb{R}, \quad x \mapsto \begin{cases}\varepsilon_{n}^{(N-2) / 2} u_{n}\left(\varepsilon_{n} x+a_{n}\right) & \text { if } x \in \Omega_{n}=\frac{\Omega-a_{n}}{\varepsilon_{n}}, \\ 0 & \text { if } x \notin \Omega_{n} .\end{cases}
$$

We have

$$
\int_{\mathbb{R}^{N}}\left|\nabla v_{n}\right|^{2}=\int_{\Omega}\left|\nabla u_{n}\right|^{2}, \quad \int_{\mathbb{R}^{N}}\left|v_{n}\right|^{p+1}=\int_{\Omega}\left|u_{n}\right|^{p+1}
$$

so we may assume that $\nabla v_{n} \rightarrow \nabla w$ in $L^{2}\left(\mathbb{R}^{N}\right), v_{n} \rightarrow w$ in $L^{p+1}\left(\mathbb{R}^{N}\right), v_{n} \rightarrow w$ a.e., $\Omega_{n} \rightarrow U$ and $w$ satisfies

$$
-\Delta w=w^{p}, w \geq 0 \quad \text { on } U ; \quad w=0 \quad \text { on } \partial U .
$$

We are in one of the three cases:

1) $\varepsilon_{n} \rightarrow l>0$

2) $\varepsilon_{n} \rightarrow 0$ and $\left(1 / \varepsilon_{n}\right) d\left(a_{n}, \partial \Omega\right) \rightarrow l<+\infty$,

3) $\varepsilon_{n} \rightarrow 0$ and $\left(1 / \varepsilon_{n}\right) d\left(a_{n}, \partial \Omega\right) \rightarrow+\infty$. 
Cases 1) and 2) cannot occur. Indeed, assume that $\varepsilon_{n} \rightarrow l>0$. We show that $\int_{\mathbb{R}^{N}}\left(v_{n}^{+}\right)^{p+1} \rightarrow 0$, a contradiction to $(21)$, since

$$
\int_{B(0,1)}\left(v_{n}^{+}\right)^{p+1}=\int_{a_{n}+\varepsilon_{n} B(0,1)}\left(u_{n}^{+}\right)^{p+1}=\nu>0 .
$$

It suffices to show that, for any $a \in \mathbb{R}^{N}$ and $\zeta \in C^{\infty}\left(\mathbb{R}^{N}, \mathbb{R}\right), \zeta \geq 0, \operatorname{supp} \zeta \subset$ $a+B(0,1)$,

$$
\int_{\mathbb{R}^{N}}\left(\zeta v_{n}^{+}\right)^{p+1} \rightarrow 0
$$

Note that, as $u_{n} \rightarrow 0$ in $H_{0}^{1}(\Omega)$, one can assume that $u_{n} \rightarrow 0$ in $L^{2}(\Omega)$, and then $\int_{\mathbb{R}^{N}} v_{n}^{2}=\left(1 / \varepsilon_{n}^{2}\right) \int_{\Omega} u_{n}^{2} \rightarrow 0 . J^{\prime}\left(u_{n}\right) \rightarrow 0$ gives us, through the rescaling (22),

$$
-\Delta v_{n}=\left(v_{n}^{+}\right)^{p}+g_{n} \quad \text { on } \Omega_{n}, \text { with } g_{n} \rightarrow 0 \text { in } H^{-1}\left(\Omega_{n}\right) .
$$

Multiplying this equation by $\zeta^{2} v_{n}$ and integrating on $\Omega_{n}$ we obtain

$$
\begin{aligned}
\int_{\Omega_{n}}\left|\nabla \zeta v_{n}^{+}\right|^{2}+o(1) & =\int_{\Omega_{n}} \zeta^{2}\left(v_{n}^{+}\right)^{p+1}+o(1) \\
& \leq\left(\int_{\sup \zeta}\left(v_{n}^{+}\right)^{p+1}\right)^{\frac{p-1}{p+1}}\left(\int_{\Omega_{n}}\left(\zeta v_{n}^{+}\right)^{p+1}\right)^{\frac{2}{p+1}}+o(1) .
\end{aligned}
$$

Then the Sobolev inequality yields

$$
S \leq \nu^{(p-1) /(p+1)}+o(1),
$$

a contradiction to (20). Concerning case 2) Pokhozhaev's identity on $U$ which is here a half-plane implies that $w \equiv 0$. Therefore $\nabla v_{n} \rightarrow 0$ in $L^{2}\left(\mathbb{R}^{N}\right), v_{n} \rightarrow 0$ in $L^{p+1}\left(\mathbb{R}^{N}\right), v_{n} \rightarrow 0$ in $L^{2}\left(\mathbb{R}^{N}\right)$, and the same argument as above shows that $v_{n} \rightarrow 0$ in $L_{\text {loc }}^{p+1}\left(\mathbb{R}^{N}\right)$, contrary to $(24)$. Hence we are in case 3$)$, and $w \not \equiv 0$ (otherwise we get a contradiction to the choice of $\nu$, as previously). It follows from (23) with $U=\mathbb{R}^{N}$ that

$$
w=\alpha_{N} \delta_{\lambda, x} \quad \text { for some } \lambda \in \mathbb{R}^{N} \text { and } x \in \mathbb{R}^{N} .
$$

We define $w_{n}$ on $\Omega$ as

$$
w_{n}(x)=\frac{1}{\varepsilon_{n}^{(N-2) / 2}} P w\left(\frac{1}{\varepsilon_{n}}\left(x-a_{n}\right)\right)
$$

and we set

$$
u_{n}^{(1)}=u_{n}-w_{n} .
$$

Then it follows from the characterization of $w_{n}$ that

(25) $\int_{\Omega}\left|\nabla u_{n}^{(1)}\right|^{2}=\int_{\Omega}\left|\nabla u_{n}\right|^{2}-\int_{\mathbb{R}^{N}}|\nabla w|^{2}+o(1)=\int_{\Omega}\left|\nabla u_{n}\right|^{2}-S_{N}^{N / 2}+o(1)$,

$$
J\left(u_{n}^{(1)}\right)=J\left(u_{n}\right)-J(w)+o(1)=J\left(u_{n}\right)-\frac{S^{N / 2}}{N}+o(1),
$$




$$
J^{\prime}\left(u_{n}^{(1)}\right) \rightarrow 0,
$$

and we can iterate the process until $J\left(u_{n}^{(l)}\right)<S^{N / 2} / N$, and thus $u_{n}^{(l)} \rightarrow 0$ in $H_{0}^{1}(\Omega)$. According to $(26)$, this happens for some $l=k$. Thus, we get

$$
u_{n}=\sum_{i=1}^{k} \alpha_{N} P \delta_{\lambda_{i}^{n}, x_{i}^{n}}+v_{n}
$$

with $v_{n} \rightarrow 0$ in $H_{0}^{1}(\Omega)$, and $\lambda_{i}^{n} d\left(x_{i}^{n}, \partial \Omega\right) \rightarrow+\infty$. Moreover, according to (25) we have

$$
\int_{\Omega}\left|\nabla u_{n}\right|^{2}=\sum_{i=1}^{k} \alpha_{N}^{2} \int_{\Omega}\left|\nabla P \delta_{\lambda_{i}^{n}, x_{i}^{n}}\right|^{2}+o(1)
$$

and this implies (13).

As a consequence of the previous arguments, we obtain a characterization of the potential critical points at infinity of $J$ :

If $\left(u_{n}\right)$ is a sequence in $H_{0}^{1}$ satisfying

$$
J\left(u_{n}\right) \rightarrow c_{k}=k S^{N / 2} / N, \quad J^{\prime}\left(u_{n}\right) \rightarrow 0,
$$

there exist sequences $\left(x_{i}^{n}\right)$ in $\Omega$ and $\left(\lambda_{i}^{n}\right)$ in $\mathbb{R}_{+}^{*}$ such that (19) holds, together with (12), (13). We may assume that, possibly for a subsequence, $x_{i}^{n}$ goes to $x_{i} \in \bar{\Omega}$ as $n$ goes to infinity. Then the set of potential critical points at infinity may be in some sense parametrized by $\bar{\Omega}^{k}$. Once we performed this representation, the question is to compute the change of topology between the level sets across the level $c_{k}$. The result obtained in [5], [6], using a deformation method and a careful analysis of $J^{\prime \prime}$ near the critical points at infinity (Morse theory at infinity), reads

$$
\left(J_{c_{k}+\varepsilon}, J_{c_{k}-\varepsilon}\right) \simeq\left(X \times D^{1},\left(X \times S^{0}\right) \cup\left(Y \times D^{1}\right)\right)
$$

where

$$
X=\Omega^{k} \underset{\sigma_{k}}{\times} D^{k-1}, \quad Y=\left(\Omega^{k} \underset{\sigma_{k}}{\times} S^{k-2}\right) \cup\left(I_{k} \underset{\sigma_{k}}{\times} D^{k-1}\right),
$$

$\sigma_{k}$ denoting the group of permutations of $\{1, \ldots, k\}$, and

$$
I_{k}=\left\{x \in \Omega^{k}: \varrho(x) \leq 0\right\}
$$

(see (5), (6) for the definition of $\varrho$ on $\Omega^{k}$ ). For $k=1$

$$
\left(J_{c_{k}+\varepsilon}, J_{c_{k}-\varepsilon}\right) \simeq\left(\Omega \times D^{1}, \Omega \times S^{0}\right) .
$$

From this computation we deduce the following lemma:

There is some $k_{0} \in \mathbb{N}^{*}$, depending only on $\Omega$, such that $\left(J_{c_{k}+\varepsilon}, J_{c_{k}-\varepsilon}\right) \simeq 0$ for every $k \geq k_{0}$.

This lemma follows from the definition of $\varrho$. If $\mathbf{x}=\left(x_{1}, \ldots, x_{k}\right) \in \Omega^{k}$ and $k$ is large, some points $x_{i}$ and $x_{j}$ are very close to each other, $G\left(x_{i}, x_{j}\right)$ goes to $-\infty$ and so $\varrho(\mathbf{x}) \leq 0$. Thus, for $k$ large enough, $I_{k}=\Omega^{k}$ and $\left(J_{c_{k}+\varepsilon}, J_{c_{k}-\varepsilon}\right) \simeq 0$ from (29). Using the topological assumption on $\Omega$, Bahri and Coron also show the following lemma: 
If $H_{2 l-1}(\Omega ; \mathbb{Q}) \neq 0$ or $H_{l}(\Omega ; \mathbb{Z} / 2 \mathbb{Z}) \neq 0$ for some $l \in \mathbb{N}^{*}$, then $\left(J_{c_{k}+\varepsilon}, J_{c_{k}-\varepsilon}\right) \not 0, \forall k \in \mathbb{N}^{*}$.

This last result is proved by induction on $k$ and requires refined arguments from algebraic topology. The contradiction between the two lemmas gives the result of Theorem 2 .

2. The subcritical approximation. In this section, I would like to explain some recent results concerning the problems

$$
\left\{\begin{array}{l}
-\Delta u=u^{p-\varepsilon}, \quad u>0 \quad \text { on } \Omega, \\
u=0 \text { on } \partial \Omega
\end{array}\right.
$$

with $\varepsilon>0$. The associated functional defined on $H_{0}^{1}(\Omega)$,

$$
J_{\varepsilon}(u)=\frac{1}{2} \int_{\Omega}|\nabla u|^{2}-\frac{1}{p+1-\varepsilon} \int_{\Omega}|u|^{p+1-\varepsilon},
$$

satisfies the Palais-Smale condition and has strictly positive critical points which are solutions to $\left(\mathrm{P}_{\varepsilon}\right)$ (see for example [20]). As $\varepsilon$ goes to zero, these solutions may either converge in $H_{0}^{1}(\Omega)$ to a solution $u_{0}$ of the limit problem $(\mathrm{P})$ (possibly $u_{0} \equiv$ 0 ), or blow up at a finite number of points of $\Omega$, under the assumption that these solutions are uniformly bounded in $H_{0}^{1}(\Omega)$. More precisely, an argument similar to the blow-up analysis carried out in the previous section shows that if $\left(u_{\varepsilon}\right)$ is an $H_{0}^{1}$ bounded sequence of solutions to $\left(\mathrm{P}_{\varepsilon}\right)$, then, possibly for a subsequence, one has

$$
u_{\varepsilon}=u_{0}+\sum_{i=1}^{k} \alpha_{i}^{\varepsilon} P \delta_{\lambda_{i}^{\varepsilon}, x_{i}^{\varepsilon}}+v^{\varepsilon}
$$

where $u_{0}$ is either a solution to $(\mathrm{P})$, or $u_{0} \equiv 0$, and if $k>0$ then

$$
\begin{gathered}
\alpha_{i}^{\varepsilon} \rightarrow \alpha_{N}, \quad \lambda_{i}^{\varepsilon} d\left(x_{i}^{\varepsilon}, \partial \Omega\right) \rightarrow+\infty, \\
\frac{\lambda_{i}^{\varepsilon}}{\lambda_{j}^{\varepsilon}}+\frac{\lambda_{j}^{\varepsilon}}{\lambda_{i}^{\varepsilon}}+\lambda_{i}^{\varepsilon} \lambda_{j}^{\varepsilon}\left|x_{i}^{\varepsilon}-x_{j}^{\varepsilon}\right|^{2} \rightarrow+\infty, \quad i \neq j,
\end{gathered}
$$

as $\varepsilon \rightarrow 0$. Moreover, we get the estimates ([30])

$$
\begin{aligned}
\left|u_{\varepsilon}\right|_{H_{0}^{1}}^{2} & =\left|u_{0}\right|_{H_{0}^{1}}^{2}+k S^{N / 2}+o(\varepsilon), \\
J\left(u_{\varepsilon}\right) & =J\left(u_{0}\right)+k \frac{S^{N / 2}}{N}\left(=c_{k}\right)+o(\varepsilon) .
\end{aligned}
$$

In fact, a new and important result of R. Schoen [29] implies that for $u_{\varepsilon}$ a solution to $\left(\mathrm{P}_{\varepsilon}\right)$ we have the alternative: either $u_{0} \equiv 0$, or $k=0$. In the following, we are interested of course in the case $k \neq 0$ (and thus $u_{0} \equiv 0$ in (31)), i.e. in the solutions to $\left(\mathrm{P}_{\varepsilon}\right)$ which blow up at $k$ points $x_{1}, \ldots, x_{k}$ of $\bar{\Omega}$ as $\varepsilon \rightarrow 0$. In a first step, we will give a precise description of the points of $\Omega$ at which such solutions concentrate. As at the same time $J_{\varepsilon}\left(u_{\varepsilon}\right) \rightarrow c_{k}$, we will then compute the difference of topology that 
they induce between the level sets of the functional across the level $c_{k}$. Before stating the results, let us introduce some notations. For $\mathbf{x} \in \Omega^{k}$, we define the function

$$
F_{\mathbf{x}}:\left(\mathbb{R}_{+}^{*}\right)^{k} \rightarrow \mathbb{R}, \quad \Lambda=\left(\Lambda_{1}, \ldots, \Lambda_{k}\right) \mapsto \frac{1}{2}^{t} \Lambda M(\mathbf{x}) \Lambda-\log \Lambda_{1} \ldots \Lambda_{k},
$$

where $M(\mathbf{x})$ is the matrix defined in (4), (5). We recall that we denote by $\varrho(\mathbf{x})$ its smallest eigenvalue. If $\varrho(\mathbf{x})>0$, then $F_{\mathbf{x}}$ is strictly convex on $\left(\mathbb{R}_{+}^{*}\right)^{k}$, infinite on the boundary of this domain, and so has in it a unique critical point $\Lambda(\mathbf{x})$ which is a minimum. On the subset $\varrho^{+}=\left\{\mathbf{x} \in \Omega^{k}: \varrho(\mathbf{x})>0\right\}$, we then define the function

$$
\widetilde{F}(\mathbf{x})=F_{\mathbf{x}}(\Lambda(\mathbf{x}))=\frac{k}{2}-\log \Lambda_{1}(\mathbf{x}) \ldots \Lambda_{k}(\mathbf{x})
$$

whose differential is given by

$$
\widetilde{F}^{\prime}(\mathbf{x})=\frac{1}{2}^{t} \Lambda(\mathbf{x}) M^{\prime}(\mathbf{x}) \Lambda(\mathbf{x})=-\sum_{i=1}^{k} \frac{\Lambda_{i}^{\prime}(\mathbf{x})}{\Lambda_{i}(\mathbf{x})} .
$$

We are now able to state the first result $([7])$ :

THEOREM 4. Assume that $N \geq 4$, and that $\left(u_{\varepsilon}\right)$ is a sequence of solutions to $\left(\mathrm{P}_{\varepsilon}\right)$ which blows up at $k$ points $x_{1}, \ldots, x_{k}$ of $\bar{\Omega}$ as $\varepsilon \rightarrow 0$ (i.e. $\left|\nabla u_{\varepsilon}\right|^{2}, u_{\varepsilon}^{p+1} \rightarrow$ $S^{N / 2} \sum_{i=1}^{k} \delta_{x_{i}}$ as $\varepsilon \rightarrow 0$ in the sense of measures). The conclusions are:

(i) $\mathbf{x}=\left(x_{1}, \ldots, x_{k}\right) \in \Omega_{d_{0}}^{k}$, where $d_{0}$ is a strictly positive constant which depends on $\Omega$ only and $\Omega_{d_{0}}=\left\{x \in \Omega: d(x, \partial \Omega)>d_{0}\right\}$.

(ii) $\varrho(\mathbf{x}) \geq 0$.

(iii) Either $\varrho(\mathbf{x})>0$ and $\widetilde{F}^{\prime}(\mathbf{x})=0$, or $\varrho(\mathbf{x})=0$ and $\varrho^{\prime}(\mathbf{x})=0$.

The index of $u_{\varepsilon}$ as a critical point of $J_{\varepsilon}$ is at least $l+k$ (and at most $\left.(N+1) k\right)$, where $l$ is the index of $\mathbf{x}$ as a critical point of $\widetilde{F}($ or $\varrho)$. If $\mathbf{x} \in \varrho^{+}$is a nondegenerate critical point of $\widetilde{F}$, the index of $u_{\varepsilon}$ is exactly $l+k$.

Conversely, if $\mathbf{x}=\left(x_{1}, \ldots, x_{n}\right) \in \varrho^{+}$is a nondegenerate critical point of $\widetilde{F}$, there exists for $\varepsilon$ small enough a sequence $\left(u_{\varepsilon}\right)$ of solutions to $\left(\mathrm{P}_{\varepsilon}\right)$ which concentrate at $x_{1}, \ldots, x_{k}$ as $\varepsilon \rightarrow 0$.

Under the assumption that 0 is not a critical level for $\varrho$ (which is at least true generically), (ii) and (iii) may be replaced by

(ii) $^{\prime} \varrho(\mathbf{x}) \geq \varrho_{0}$, where $\varrho_{0}$ is a strictly positive constant which depends on $\Omega$ only.

(iii) ${ }^{\prime} \widetilde{F}^{\prime}(\mathbf{x})=0$.

Morever, we have the estimates

$$
\frac{1}{\left(\lambda_{i}^{\varepsilon}\right)^{(N-2) / 2}} \sim \gamma \Lambda_{i}(x) \varepsilon^{1 / 2}
$$

and

$$
J_{\varepsilon}\left(u_{\varepsilon}\right)=c_{k}+k \gamma_{1} \varepsilon|\log \varepsilon|+k \gamma_{2} \varepsilon+2 \gamma_{1} \varepsilon \widetilde{F}(\mathbf{x})+o(\varepsilon)
$$

where $\gamma>0, \gamma_{1}>0, \gamma_{2}$ are constants which depend on $N$ only. 
R e marks. 1. R. Schoen had already shown in [29] that all the concentrations were simple, and that the speeds of concentration $\lambda_{i}^{\varepsilon}$ were of the same order. (i)(ii) also imply that the blow-up points $x_{1}, \ldots, x_{k}$ have to be far from each other: $\exists d_{0}^{\prime}>0, \forall i, j, i \neq j,\left|x_{i}-x_{j}\right|>d_{0}^{\prime}$. This condition cannot be satisfied if $k$ is too large.

2. In the case $k=1, M(\mathbf{x})=H(x, x)>0$ everywhere, $\Lambda(\mathbf{x})=H(x, x)^{-1 / 2}$, $\widetilde{F}(\mathbf{x})=\frac{1}{2}+\frac{1}{2} \log H(x, x)$.

3 . In the case $k=2, \mathbf{x}=\left(x_{1}, x_{2}\right)$,

$$
\begin{gathered}
M(\mathbf{x})=\left(\begin{array}{cc}
H\left(x_{1}, x_{1}\right) & G\left(x_{1}, x_{2}\right) \\
G\left(x_{1}, x_{2}\right) & H\left(x_{2}, x_{2}\right)
\end{array}\right), \\
\Lambda_{1}^{2}(\mathbf{x})=\frac{H\left(x_{2}, x_{2}\right)^{1 / 2}}{H\left(x_{1}, x_{2}\right)^{1 / 2}} \frac{1}{\phi(\mathbf{x})}, \quad \widetilde{F}(\mathbf{x})=1+\log \phi(\mathbf{x}),
\end{gathered}
$$

with

$$
\phi(\mathbf{x})=H\left(x_{1}, x_{1}\right)^{1 / 2} H\left(x_{2}, x_{2}\right)^{1 / 2}-G\left(x_{1}, x_{2}\right) .
$$

As

$\varrho(\mathbf{x})=\frac{1}{2}\left[H\left(x_{1}, x_{1}\right)+H\left(x_{2}, x_{2}\right)-\left(\left(H\left(x_{1}, x_{1}\right)-H\left(x_{2}, x_{2}\right)\right)^{2}+4 G\left(x_{1}, x_{2}\right)^{2}\right)^{1 / 2}\right]$, $\varrho(\mathbf{x})>0$ is equivalent to $\phi(\mathbf{x})>0$ and one can replace (ii) and (iii) in the theorem by $\phi(\mathbf{x}) \geq 0, \phi^{\prime}(\mathbf{x})=0$ (since for $\varrho=0$ we also have $\varrho^{\prime}=0 \Leftrightarrow \phi^{\prime}=0$ ). This result was proved in [27].

In order to prove Theorem 4, one proceeds as follows:

1. Consider the set

$U=\left\{\sum_{i=1}^{k} \alpha_{N} P \delta_{\lambda_{i}, x_{i}}: \lambda_{i} d\left(x_{i}, \partial \Omega\right)>m_{0} ; \frac{\lambda_{i}}{\lambda_{j}}+\frac{\lambda_{j}}{\lambda_{i}}+\lambda_{i} \lambda_{j}\left|x_{i}-x_{j}\right|^{2}>m_{0}, i \neq j\right\}$,

where $m_{0}$ is some large constant. For $u \in H_{0}^{1}(\Omega)$ close to $U$, the problem

$$
\text { Minimize }\left|u-\sum_{i=1}^{k} \alpha_{i} P \delta_{\lambda_{i}, x_{i}}\right|_{H_{0}^{1}}
$$

with respect to $\alpha_{i}, \lambda_{i}, x_{i}$ has a unique solution up to permutations ([6]). Then one establishes a diffeomorphism between a neighborhood of the possible singular solutions of $\left(\mathrm{P}_{\varepsilon}\right)$ we are interested in (which is also a neighborhood of the potential critical points at infinity for $(\mathrm{P})$ ), and the manifold

$$
\begin{gathered}
M=\left\{(\alpha, \lambda, \mathbf{x}, v) \in \mathbb{R}^{k} \underset{\sigma_{k}}{\times}\left(\mathbb{R}_{+}^{*}\right)^{k} \underset{\sigma_{k}}{\times} \Omega^{k} \times H_{0}^{1}(\Omega):\left|\alpha_{i}-\alpha_{N}\right|<\nu_{0} ;\right. \\
\lambda_{i} d\left(x_{i}, \partial \Omega\right)>n_{0} ; \frac{\lambda_{i}}{\lambda_{j}}+\frac{\lambda_{j}}{\lambda_{i}}+\lambda_{i} \lambda_{j}\left|x_{i}-x_{j}\right|^{2}>n_{0}, i \neq j ; \\
\left.|v|_{H_{0}^{1}}<\kappa_{0}, v \in E_{\lambda, \mathbf{x}}\right\}
\end{gathered}
$$


where $\nu_{0}, \kappa_{0}$ are small constants and $n_{0}$ a large one, and

$$
\begin{aligned}
E_{\lambda, \mathbf{x}}=\left\{v \in H_{0}^{1}(\Omega):\left\langle v, P \delta_{\lambda_{i}, x_{i}}\right\rangle_{H_{0}^{1}}\right. & =\left\langle v, \frac{\partial P \delta_{\lambda_{i}, x_{i}}}{\partial \lambda_{i}}\right\rangle_{H_{0}^{1}} \\
& \left.=\left\langle v, \frac{\partial P \delta_{\lambda_{i}, x_{i}}}{\partial x_{i}}\right\rangle_{H_{0}^{1}}=0, \forall_{i}\right\} .
\end{aligned}
$$

Then $u=\sum_{i=1}^{k} \alpha_{i} P \delta_{\lambda_{i}, x_{i}}+v$ is a critical point of $J_{\varepsilon}$ if and only if $(\alpha, \lambda, \mathbf{x}, v)$ is a critical point of the functional $K_{\varepsilon}(\alpha, \lambda, \mathbf{x}, v)=J_{\varepsilon}(u)$ on $M$.

2. The vanishing of the partial derivatives of $K_{\varepsilon}$ on $M$ provides us with equations whose resolution gives us the results of Theorem 4 . The proof requires careful estimates that we will not make explicit here.

- The equation relative to $\partial K_{\varepsilon} / \partial v$ proves that $v$ is very small in $H_{0}^{1}$-norm, so that the $v$-part is negligible for all practical purposes (at the possible exception of dimension 3 in which precisely we cannot conclude).

- The equation relative to $\partial K_{\varepsilon} / \partial \alpha$ proves that each $\alpha_{i}$ is very close to $\alpha_{N}$, and may even be made equal to $\alpha_{N}$ in all practical computations.

- The equation relative to $\partial K_{\varepsilon} / \partial \lambda$ implies that $\varrho(\mathbf{x}) \geq 0$ and the estimate (35) in the case $\varrho(\mathbf{x})>0$.

- Lastly, the equation relative to $\partial K_{\varepsilon} / \partial \mathbf{x}$ shows that $\widetilde{F}^{\prime}(\mathbf{x})=0$ in the case $\varrho(\mathbf{x})>0$, and $\varrho^{\prime}(\mathbf{x})=0$ if $\varrho(\mathbf{x})=0$.

3. A critical point $(\alpha, \lambda, \mathbf{x}, v) \in M$ of $K_{\varepsilon}$ may be obtained by successive optimizations. $v$ corresponds to a minimum (index 0 ), $\alpha$ to a maximum (index $k), \lambda$ to a minimum (index 0 ), $\mathbf{x}$ is a critical point of a perturbation of $\widetilde{F}$ (or $\varrho$ ), hence a contribution to the global index of the solution which is between $l$ and $N k$, where $l$ is the index of $\mathbf{x}$ as a critical point of $\widetilde{F}$ (or $\varrho$ ), and which is exactly $l$ in case of nondegeneracy.

Once Theorem 4 is proved, we can take advantage of the precise characterization of the $k$-singular solutions of $\left(\mathrm{P}_{\varepsilon}\right)$ to define a smallest neighborhood $V$ (of the corresponding critical points of $K_{\varepsilon}$ ) than $M$, such that on the boundary of $V$, either $-K_{\varepsilon}^{\prime}$ is pointing inward $V$, or $K_{\varepsilon}$ is below the least critical value on $M$.

Our aim being now to compute the contribution of the $k$-singular solutions of $\left(\mathrm{P}_{\varepsilon}\right)$ to the relative topology $\left(J_{\varepsilon}^{c_{k}+\delta}, J_{\varepsilon}^{c_{k}-\delta}\right)$, we are reduced to study the relative topology $\left(K_{\varepsilon}^{c_{k}+\delta} \cap V, K_{\varepsilon}^{c_{k}-\delta} \cap V\right)$. Proceeding as previously by successive optimizations, we get ([7])

Theorem 5. Assume that $N \geq 4$ and 0 is not a critical value for $\varrho$. The contribution to the relative topology of the level set

$$
J_{\varepsilon}^{c_{k}+\delta}=\left\{u \in H_{0}^{1}(\Omega): J_{\varepsilon}(u) \leq c_{k}+\delta\right\}
$$

with respect to the level set

$$
J_{\varepsilon}^{c_{k}-\delta}=\left\{u \in H_{0}^{1}(\Omega): J_{\varepsilon}(u) \leq c_{k}-\delta\right\}
$$


of the possible solutions of $\left(\mathrm{P}_{\varepsilon}\right)$ which blow up at $k$ points as $\varepsilon$ goes to zero is equal for $\varepsilon$ small enough to the relative topology

$$
\left(\Omega^{k}, \varrho^{-}\right) \underset{\sigma_{k}}{\times}\left(D^{k}, S^{k-1}\right)
$$

with $\varrho^{-}=\left\{\mathbf{x} \in \Omega^{k}: \varrho(\mathbf{x}) \leq 0\right\}$.

This result points out the importance of the behavior of the least eigenvalue $\varrho(\mathbf{x})$ of the matrix $M(\mathbf{x})$ on $\Omega^{k}$, which was already noted in [3], [5], [6] (see also Theorem 3).

Note that the difference of topology between the level sets of $J_{\varepsilon}$ may only come from the existence of critical points, since the functionals satisfy the Palais-Smale condition. Theorem 5 shows that if the relative topology $\left(\Omega^{k}, \varrho^{-}\right)$is nontrivial, $\left(\mathrm{P}_{\varepsilon}\right)$ has solutions which blow up at $k$ points as $\varepsilon$ goes to zero, without any nondegeneracy assumption as in Theorem 4.

The stability with respect to $\varepsilon$ of the result that we obtained implies, through some arguments of algebraic topology, that the relative homology that we computed is exactly the contribution of the critical points at infinity to the relative homology between the level sets of the functional $J$ across the level $c_{k}$. Then, by coming back to the arguments developed in Section 1, this result should enable us to answer in the future the questions concerning existence and multiplicity of solutions to $(\mathrm{P})$ raised by $\mathrm{R}$. Bott.

\section{References}

[1] T. Aubin, Problèmes isopérimétriques et espaces de Sobolev, J. Differential Geom. 11 (1976), 573-598.

[2] - Equations différentielles non linéaires et problème de Yamabe concernant la courbure scalaire, J. Math. Pures Appl. 55 (1976), 269-296.

[3] A. Bahri, Critical Points at Infinity in Some Variational Problems, Pitman Res. Notes Math. Ser. 182, Longman, 1989.

[4] A. Bahri et J. M. Coron, Vers une théorie des points critiques à l'infini, Séminaire EDP Ecole Polytechnique 1984-1985, n 8 .

[5] - - - Sur une équation elliptique non linéaire avec l'exposant critique de Sobolev, C. R. Acad. Sci. Paris Sér. I 301 (1985), 345-348.

[6] - - - On a nonlinear elliptic equation involving the critical Sobolev exponent: the effect of the topology of the domain, Comm. Pure Appl. Math. 41 (1988), 255-294.

[7] A. Bahri, Y. Li and O. Rey, On a variational problem with lack of compactness. The topological effect of the critical points at infinity, to appear.

[8] H. Brézis and J. M. Coron, Convergence of solutions of H-systems or how to blow bubbles, Arch. Rational Mech. Anal. 89 (1985), 21-56.

[9] H. Brézis and L. Nirenberg, Positive solutions of nonlinear elliptic equations involving critical Sobolev exponents, Comm. Pure Appl. Math. 36 (1983), 437-477.

[10] H. Brézis and L. A. Peletier, Asymptotics for elliptic equations involving the critical growth, in: Partial Differential Equations and the Calculus of Variations, F. Colombani and S. Spagnolo (eds.), Birkhäuser, 1989. 
[11] J. M. Coron, Topologie et cas limite des injections de Sobolev, C. R. Acad. Sci. Paris Sér. I 299 (1984), 209-212.

[12] E. N. Dancer, A note on an equation with critical exponent, Bull. London Math. Soc. 20 (1988), 600-602.

[13] W. Y. Ding, On a conformally invariant elliptic equation on $\mathbb{R}^{n}$, Comm. Math. Phys. 107 (1986), 331-335.

[14] - Positive solutions of $\Delta u+u^{(n+2) /(n-2)}=0$ on contractible domains, to appear.

[15] B. Gidas, Symmetry properties and isolated singularities of positive solutions of nonlinear elliptic equations, in: Nonlinear Partial Differential Equations in Engineering and Applied Science, Sternberg et al. (eds.), Dekker, New York 1980, 255-273.

[16] B. Gidas, W.-M. Ni and L. Nirenberg, Symmetry of positive solutions of nonlinear elliptic equations in $\mathbb{R}^{n}$, in: Mathematical Analysis and Applications, Part A, L. Nachbin (ed.), Academic Press, 1981, 370-402.

[17] Z. C. Han, Asymptotic approach to singular solutions for nonlinear elliptic equations involving critical Sobolev exponent, Ann. Inst. Poincaré Anal. Non Linéaire 8 (1991), 159174 .

[18] J. Kazdan, Prescribing the Curvature of a Riemannian Manifold, CBMS Regional Conf. Ser. in Math. 57, Amer. Math. Soc., 1985.

[19] E. Lieb, Sharp constants in the Hardy-Littlewood-Sobolev and related inequalities, Ann. of Math. 118 (1983), 349-374.

[20] P. L. Lions, The concentration-compactness principle in the Calculus of Variations. The locally compact case. Parts 1 and 2, Ann. Inst. Poincaré Anal. Non Linéaire 1 (1984), 109-145 and 223-284.

[21] - , The concentration-compactness principle in the calculus of variations. The limit case, Rev. Mat. Iberoamericana 1(1) (1985), 145-201, and 1(2) (1985), 45-121.

[22] M. Obata, The conjectures on conformal transformations of Riemannian manifolds, J. Differential Geom. 6 (1971), 247-258.

[23] S. I. Pokhozhaev, Eigenfunctions of the equation $\Delta u+\lambda f(u)=0$, Soviet Math. Dokl. 6 (1965), 1408-1411.

[24] O. Rey, The role of the Green's function in a nonlinear elliptic equation involving the critical Sobolev exponent, J. Funct. Anal. 89 (1990), 1-52.

[25] - Sur un problème variationnel non compact: l'effet de petits trous dans le domaine, C. R. Acad. Sci. Paris Sér. I 308 (1989), 349-352.

[26] - The proof of two conjectures of H. Brézis and L. A. Peletier, Manuscripta Math. 65 (1989), 19-37.

[27] - Blow-up points of solutions to elliptic equations with limiting non linearity, Differential and Integral Equations, to appear.

[28] R. Schoen, Conformal deformation of a Riemannian metric to constant scalar curvature, J. Differential Geom. 20 (1984), 479-495.

[29] - in preparation.

[30] M. Struwe, A global compactness result for elliptic boundary value problems involving limiting nonlinearities, Math. Z. 187 (1984), 511-517.

[31] G. Talenti, Best constant in Sobolev inequality, Ann. Mat. Pura Appl. 110 (1976), 353372 .

[32] N. Trudinger, Remarks concerning the conformal deformation of Riemannian structures on compact manifolds, Ann. Scuola Norm. Sup. Pisa 22 (1968), 265-274.

[33] H. Yamabe, On a deformation of Riemannian structures on compact manifolds, Osaka Math. J. 12 (1960), 21-37. 